\title{
Patterning, integration and characterisation of polymer optical oxygen sensors for microfluidic devices $\dagger$
}

\author{
Volker Nock, ${ }^{* a}$ Richard J. Blaikie $^{a}$ and Tim David ${ }^{b}$ \\ Received 4th February 2008, Accepted 6th June 2008 \\ First published as an Advance Article on the web 23rd June 2008 \\ DOI: $10.1039 / \mathrm{b801879k}$
}

This paper describes a process for the layer-by-layer fabrication and integration of luminescent dye-based optical oxygen sensors into microfluidic devices. Application of oxygen-sensitive platinum(II) octaethylporphyrin ketone fluorescent dye dissolved in polystyrene onto glass substrates by spin-coating was studied. Soft lithography with polydimethylsiloxane (PDMS) stamps and reactive ion etching in oxygen plasma were used to produce sensor patterns with a minimum feature size of $25 \mu \mathrm{m}$. Sensors patterns were integrated into a PDMS microfluidic device by plasma bonding. No degradation of the sensor response as a result of the lithography and pattern-transfer processes was detected. Gaseous and dissolved oxygen (DO) detection was characterised using fluorescence microscopy. The intensity signal ratio of the sensor films was found to increase almost two-fold from 3.6 to 6.8 by reducing film thickness from $1.3 \mu \mathrm{m}$ to $0.6 \mu \mathrm{m}$. Calibration of DO measurement showed linear Stern-Volmer behaviour that was constant for flow rates from 0.5 to $2 \mathrm{~mL} \mathrm{~min}^{-1}$. The calibrated sensors were subsequently used to demonstrate laterally resolved detection of oxygen inside a microfluidic channel. The fabrication process provides a novel, easy to use method for the repeatable integration of optical oxygen sensors into cell-culture and lab-on-a-chip devices.

\section{Introduction}

Oxygen concentration is increasingly recognized as a central parameter in cellular studies. In a natural environment, such as in mammalian organs, cellular oxygen concentration is maintained to normoxic $\left(12 \%\right.$ to $\left.\sim 0.5 \% \mathrm{O}_{2}\right)$ conditions. Regulation to within this relatively narrow range of normoxia is necessary in-vivo to prevent oxidative damage to the cell from excess oxygen (hyperoxia) and metabolic demise from insufficient oxygen (hypoxia). ${ }^{1}$

Absolute normoxic values for a specific cell are furthermore dependent on the cell localization within a particular organ. For example, in the liver, localization is exhibited in the form of oxygen modulated zonation, and gradients along the length of the sinusoid result in regionally dominant metabolic and detoxification functions. ${ }^{2}$ This natural sensitivity to local oxygen concentration can be replicated in-vitro to selectively increase specific liver cell function like urea synthesis. ${ }^{3}$ However, recent research has indicated that in-vitro cell-culture experiments performed in air $\left(\sim 21 \% \mathrm{O}_{2}\right)$ may introduce excessive stress on cells due to exposure to unnaturally high oxygen concentrations. ${ }^{4}$

${ }^{a}$ MacDiarmid Institute for Advanced Materials and Nanotechnology, Department of Electrical and Computer Engineering, University of Canterbury, Private Bag 4800, Christchurch, New Zealand.

E-mail: volker.nock@elec.canterbury.ac.nz; Fax: +64 3364 2761;

Tel: +64 33642987 ext. 7123

${ }^{b}$ Centre for Bioengineering, Department of Mechanical Engineering, University of Canterbury, Christchurch, New Zealand

$\dagger$ Electronic supplementary information (ESI) available: Micrograph of a PDMS stamp and corresponding PtOEPK/PS pattern in NIR. See DOI: $10.1039 / \mathrm{b} 801879 \mathrm{k}$
Fibroblasts exposed to different oxygen concentrations were found to adjust to high oxygen levels by reversible growth inhibition and differentiation. For neural and other stem cells in comparison, hypoxic conditions were observed to promote growth and influence differentiation. ${ }^{5}$ Measurement of oxygen concentration is therefore of special importance when comparing in-vitro results to cell behaviour observed in-vivo.

In general, oxygen uptake of cells is a powerful marker for their metabolic status, health and response to exogenous and endogenous stimuli. ${ }^{6}$ Beyond cell-culture, oxygen consumption characteristics can further be used to determine the development status of higher organisms. For example, a direct link exists between oxygen consumption of pre-implantation mammalian embryos and their development status. This indicates that integrated oxygen detection has the potential to provide biomedical applications and the agricultural industry with a reliable, non-intrusive tool to investigate embryogenesis, gene manipulation, cloning, assisted reproduction and transgenic animals. $^{7}$

However, traditional laboratory procedures for the measurement of oxygen in solution require the extraction of a sample volume for external analysis. This approach is limited by the difficulty and time needed for extraction and analysis. With fluid volumes in the range of microlitres and below, as found in current lab-on-a-chip (LOC) devices, analyte sampling constitutes a major disturbance of the system to be measured. This is a particular problem for sensing of less stable solutes such as oxygen, where retrieval is likely to significantly alter the sample. ${ }^{8}$ Alternative in-situ sensor technologies are therefore needed and we report on the patterning, integration and characterisation of such a sensor. 


\section{Integrated oxygen sensing}

Two main technologies, amperiometric electrochemical and optical sensing currently constitute the bulk of integrated sensors for the measurement of dissolved oxygen (DO) in biological applications. Both are tolerant to liquid exposure and exhibit the high sensitivity needed to detect the small changes of oxygen encountered in the cellular environment. The first principle, amperiometric electrochemical sensing using solidstate electrodes, has been applied in a variety of biomedical LOC devices. ${ }^{7,9,10}$ Despite continuous interest, several significant limitations have been identified concerning the use of amperiometric sensors. When exposed to organic matter, sensor lifetime has been found to decrease through membrane fouling, while additional problems are posed by miniaturization itself, mainly due to the need for a reference electrode and the depletion of analyte by the underlying sensing reaction. ${ }^{8}$

By contrast, fluorescent dye-based optical sensing does not exhibit analyte depletion, and has therefore emerged as a promising alternative in biomedical applications. While oxygen quenching is exhibited by the majority of fluorescent dyes, a smaller subgroup has been found to be especially suited and is used in solution ${ }^{6,11,12}$ or immobilized on a support matrix ${ }^{8,13-16}$ for the detection of DO. For use with low-cost LOCs, dye immobilization has the advantages of increasing the ease of handling, reducing the amount of fluorescent dye required and protecting the dye from potential interfering compounds. ${ }^{17}$ Platinum(II) octaethylporphyrin ketone (PtOEPK) suspended in a microporous polystyrene (PS) matrix has been identified as well suited for use in LOC devices due to its desirable optical properties and compatibility with standard optical components. PtOEPK itself is considered safe for use with biological material and has been applied to food packaging ${ }^{11}$ and in direct contact with cells in culture. ${ }^{18}$ It exhibits both a long wavelength shift and an extended long-term photostability compared to other fluorescent dyes. ${ }^{13,19}$ This allows for the use of standard optical filters and makes sample handling less critical. PS was chosen as polymer matrix because it provides good oxygen permeability, bio-compatibility and low autofluorescence.

Beyond the advantages mentioned above, the PtOEPK/PS system shares one major limitation with most other fluorescencebased sensor materials, namely the challenge to fabricate microscale patterns and integrate them with commonly used polydimethylsiloxane (PDMS) devices. Organic solvents such as acetone readily dissolve PS, making it impossible to apply photoresist on PtOEPK/PS films for standard lithographic patterning. In experiments performed with conventional positive tone resist (AZ 1518, Microchemicals, Ulm, Germany) poor wetting of the resist on the PS film was observed..$^{20}$ Furthermore, removal of the photoresist with acetone after exposure and development lead to the destruction of the PtOEPK/PS film surface. Photoresist lift-off in combination with pipetting of individual sensor patches has been demonstrated, ${ }^{13}$ but is potentially limited in throughput and minimum feature size due to the manual handling required. Previously we have suggested the use of spin-coating and dry-etching to enable wafer-level processing of polymer-encapsulated fluorescent-dye sensors. ${ }^{21}$ Seamless integration into the multilayer fabrication process, as typically encountered for microfluidic devices, ${ }^{22-24}$ was demonstrated for sensors patterned using this process. ${ }^{21}$

In this work, we show the characterisation of the fabrication process, optimization of sensor sensitivity and application to simultaneous fabrication of multiple complex sensor patterns. Performance of the integrated sensors for dissolved oxygen measurement is characterised and their application to visualise flows of different oxygen concentrations within a microfluidic channel is demonstrated. The work described has the potential to increase oxygen sensor sensitivity and enable the simple integration of complex oxygen sensor patterns in LOCs.

\section{Design}

The initial motivation for developing the sensor fabrication process stems from our previous work on the influence of microfluidic channel geometry on solute transport. ${ }^{25}$ Tapered channels can be used to produce a linearly increasing shear-stress gradient and a relatively constant oxygen concentration without the need for additional integrated gas supply. ${ }^{21}$ Sensor patterns were designed to be integrated into a PDMS microfluidic device for comparison of this particular bioreactor shape with other geometries. The device incorporates a standard rectangular parallel-plate bioreactor with constant shear-stress, the linearly increasing shear-stress shape and a tapered geometry as an intermediate state. This device acts as an example of sensor integration and will be used in the future to compare the effect of geometry on species transport.

\section{Methods}

Spin-coating and dry-etching with PDMS stamps was used to pattern fluorescence-based oxygen sensors. Custom patterns were transferred into the polymer sensor layer by reactive ion etching (RIE) and integrated with the fluidic parts by oxygen plasma bonding. High-resolution transparencies were used as photomasks for all photo-lithographical steps. Fig. 1 shows a schematic of the main fabrication steps.

\section{Chemicals and materials}

PtOEPK was purchased from Frontier Scientific (Logan, UT, USA), polystyrene (average molecular weight, $\mathrm{M}_{\mathrm{W}} 280000$ ) was purchased from Sigma-Aldrich (Auckland, NZ), photoresist (SU-8 2100) was purchased from Microchem (Newton, MA, USA), SU-8 developer ((1-methoxy-2-propyl)acetate) was purchased from Merck (Darmstadt, Germany), PDMS pre-polymer kit (Sylgard 184) was purchased from Electropar (Manukau, NZ), gases (oxygen-free nitrogen and industrial grade oxygen) were supplied by BOC (Auckland, NZ).

\section{Fabrication of the fluidic part}

Microfluidic channels were formed in PDMS by replica molding (Fig. 1(a,b)). ${ }^{26}$ The master with an inverse pattern of the channels was fabricated using SU-8 2100 negative tone photoresist on a $100 \mathrm{~mm}$ silicon wafer. First, SU-8 was applied by spin-coating to yield a $200 \mu \mathrm{m}$ thick film. After a $2 \mathrm{~h}$ solvent removal bake at $95{ }^{\circ} \mathrm{C}$ on a hotplate, the resist was exposed in a Süss MA6 mask aligner. This was followed by a post exposure bake of 


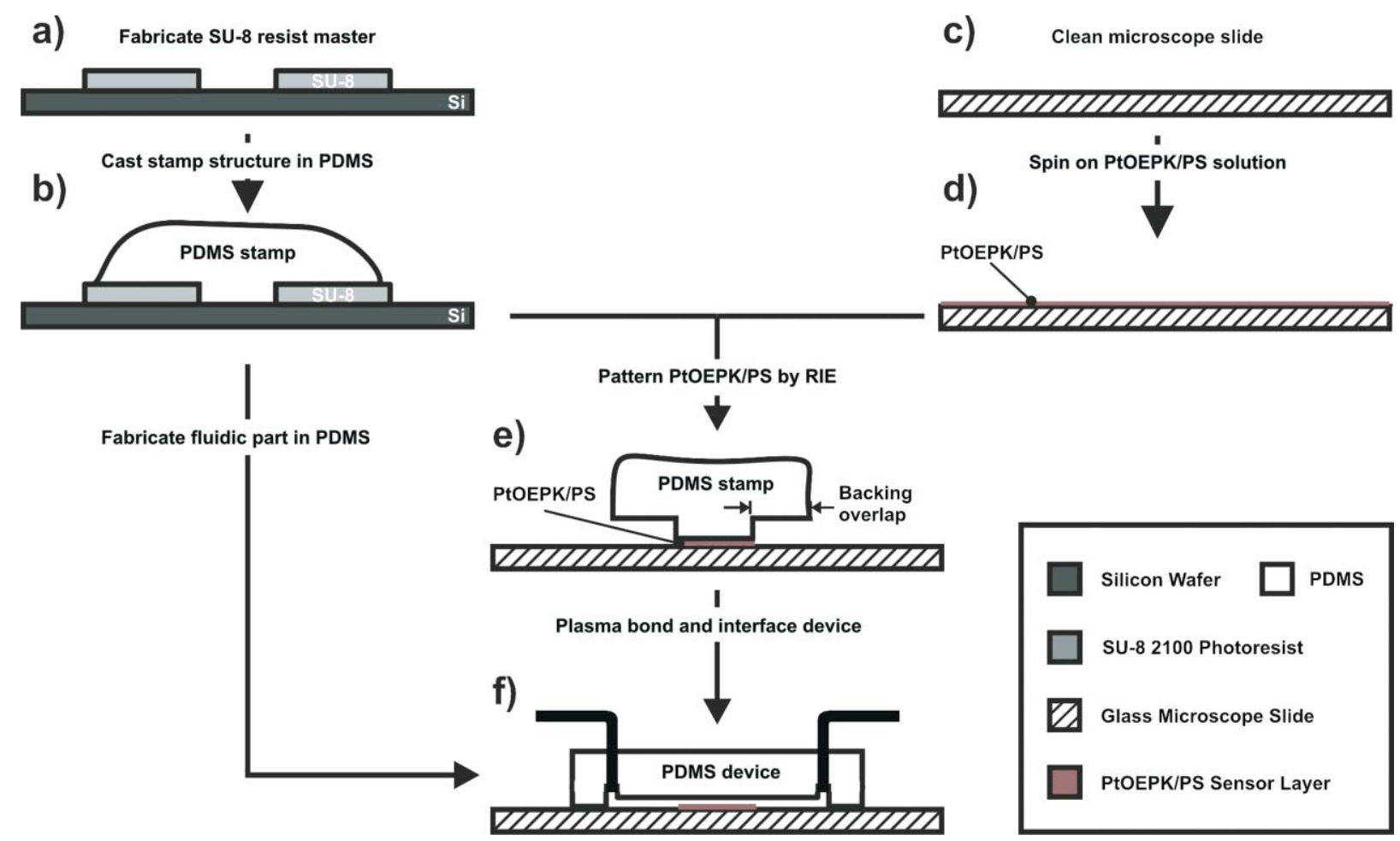

Fig. 1 Schematic of the sensor fabrication process depicting stamp fabrication in PDMS (a,b), spin-coating of the sensor layer (c,d), pattern transfer into the sensor layer by RIE (e), and integration with a PDMS microfluidic device by plasma bonding (f).

$40 \mathrm{~min}$ at $95{ }^{\circ} \mathrm{C}$ on a hotplate to activate resist cross-linking. Finally, development of the SU-8 was performed by immersion in (1-methoxy-2-propyl)acetate and patterns were rinsed with isopropanol and blow-dried with nitrogen. To facilitate removal of the cured PDMS, a fluoropolymer layer was deposited onto the resist mould. Meanwhile, liquid PDMS pre-polymer was prepared by thoroughly mixing $10: 1$ (w/w) base/curing agent. Prior to use the mixture was degassed in vacuum to remove any trapped air bubbles. The pre-polymer was then poured onto the photoresist master and cured on a hotplate at $80{ }^{\circ} \mathrm{C}$ for $90 \mathrm{~min}$. Once cured the PDMS replica was carefully peeled off and cut to size using a scalpel before being bonded to the glass substrate with the sensor layer.

\section{Spin-coating of the sensor layer}

For sensor deposition PS pellets were dissolved in toluene to yield a 7\% w/w solution. PtOEPK dye was added at $1 \mathrm{mg}$ per $1 \mathrm{~mL}$ of PS solution. Oxygen-permeable films of the $\mathrm{PtOEPK} / \mathrm{PS}$ sensor material were prepared by pipetting $200 \mu \mathrm{L}$ of the solution onto $50 \mathrm{~mm} \times 75 \mathrm{~mm}$ glass microscope slides and spinning for $30 \mathrm{~s}$ at different speeds (Fig. 1(c,d)). The thickness of the PtOEPK/PS layer was measured using a surface profilometer (Dektak, Veeco, Woodbury, NY, USA) at least $24 \mathrm{~h}$ after spin-coating.

\section{Patterning of the sensor layer}

As previously described, ${ }^{20}$ and depending on the desired pattern, PDMS stamps can be formed as negative (stencil) or positive stamps, the latter supported by a thick, overlapping backing (Fig. 1(e)). First a master mould with a positive image of the desired pattern was created in SU-8 2100 photoresist on a silicon wafer (Fig. 1(a)). PDMS pre-polymer was then poured onto the master, cured and peeled off (Fig. 1(b)). Individual stamps were cut out using a scalpel. For sensor patterning, the PDMS stamp was brought into conformal contact with the PtOEPK/PS layer. The stack was then placed in an Oxford Plasmalab 80Plus reactive ion etcher and the layer was etched using oxygen as the reactive species (Fig. 1(e)). The etching conditions used were $50 \mathrm{sccm} \mathrm{O}_{2}$ flow rate, 0.55 Torr etch pressure and $200 \mathrm{~W} \mathrm{RF}$ power applied for a typical etching time of $5 \mathrm{~min}$. Dry-etching conditions were adapted from data published for films of pure PS. $^{27}$ After plasma etching, PDMS stamps were peeled off and could be re-used. Prior to use, sensors were stored in the dark to prevent photobleaching. Partial images of the PtOEPK/PS sensor patterns were recorded using reflected light differential interference contrast (DIC) on a Nikon Eclipse 80i microscope and stitched on a PC running the Autostitch software package. ${ }^{28}$

\section{Device integration}

Assembly of the final microfluidic device for flow testing was performed by oxygen plasma bonding (Fig. 1(f)). Both, the surface of the PDMS channel network and the glass substrate with the sensor pattern, were activated in the RIE using oxygen plasma, manually aligned and brought into conformal contact. Surface activation conditions used were $50 \mathrm{sccm} \mathrm{O}_{2}$ flow rate, 75 mTorr etch pressure and $70 \mathrm{~W}$ RF power for a duration of $10 \mathrm{s.}^{23}$ A blunt needle was used to cut access ports and external fluidic interfacing was provided by insertion of Microtight tubing sleeves (Upchurch, Oak Harbour, WA, USA). 


\section{Sensor characterisation}

Sensor calibration was performed using a Nikon Eclipse 80i fluorescence microscope in combination with a digital camera (Sony Digital Handycam) in "Nightshot" mode for image acquisition. The PtOEPK dye exhibits an absorption peak at $590 \mathrm{~nm}$ and an emission peak at $760 \mathrm{~nm}$ in the near infrared (NIR), making it ideal for use with Si-based photo detectors. A special filter combination with excitation/emission filters at $595 / 760 \mathrm{~nm}$ and a $620 \mathrm{~nm}$ dichroic mirror was purchased from Chroma (Rockingham, VT, USA).

For gaseous sensor characterisation, patterns were exposed to industrial grade oxygen, oxygen free nitrogen and air. Single images were recorded after equilibration and the change of sensor intensity was analyzed using the image processing module of Matlab (Mathworks, Natick, MA, USA). First, a background image was subtracted from each image. Then, intensity was averaged over a region of interest, and compared to a reference intensity (nitrogen saturated, $0 \% \mathrm{O}_{2}$ ). To characterise the sensor patterns for DO measurement, water of defined oxygen concentration was flowed through the microfluidic device containing the sensor patterns. A flow-through oxygenator was built using gas-permeable tubing (Silastic, Dow Corning, Midland, MI, USA) in a Pyrex container. ${ }^{29}$ De-ionized (DI) water was flowed through the oxygenator and the container was perfused with different mixtures of $\mathrm{O}_{2}$ and $\mathrm{N}_{2}$, yielding a maximum dissolved oxygen concentration of $18.4 \mathrm{mg} \mathrm{L}^{-1}$. A syringe pump (PHD 2000, Harvard Apparatus, Holliston, MA, USA) was used to provide different flow rates. DO levels after the oxygenator and before the device containing the PtOEPK/PS sensor pattern were monitored via a calibrated Clarke-microelectrode reference sensor (DO-166FT, Lazar Research, Los Angeles, CA, USA). The temperature of the microfluidic chip under test was held constant at $37^{\circ} \mathrm{C}$ (cell-culture conditions) by use of a microscope warm stage (LEC Instruments, Scoresby, Australia). Images were recorded and analyzed as described above for gaseous oxygen. For the dual flow experiments the procedure was modified by use of a further identical oxygenator and a second syringe to provide two flows of different oxygen concentrations, but with equal flow rate.

\section{Results and discussion}

\section{Sensor spin-coating}

Left at room temperature (RT), spin-coated PtOEPK/PS forms solid films through evaporation of toluene. We have used two different dye solutions on a standard spin-coater to investigate the formation of PtOEPK/PS films on glass microscope slides. Glass was chosen as substrate because the low diffusivity of oxygen increases sensor stability and sensitivity. Furthermore, the ability to form an irreversible bond with PDMS makes glass the substrate of choice for LOCs. For sensor films applied by spin-coating the thickness was found to decrease linearly with increasing spin speeds. Optical inspection of the samples showed the formation of smooth, continuous films on the glass substrate with high thickness uniformity and repeatability. A quantitative test by blowing pressurized nitrogen onto the films demonstrated good adherence of the sensor without the need of increasing film adhesion by pre-etching the substrate with hydrofluoric acid, as necessary for pipetted sensor patches. ${ }^{13}$ Fig. 2 shows a plot of the film thickness obtained for two different sensor solutions as a function of spin speed. The $5 \% \mathrm{w} / \mathrm{w}$ solution of PtOEPK/PS corresponds to previously published data $^{21}$ for standard grade PS with a $\mathrm{M}_{\mathrm{W}}$ of 200000 , whereas the $7 \% \mathrm{w} / \mathrm{w}$ solution uses lower grade PS with an average $\mathrm{M}_{\mathrm{W}}$ of 280000 .

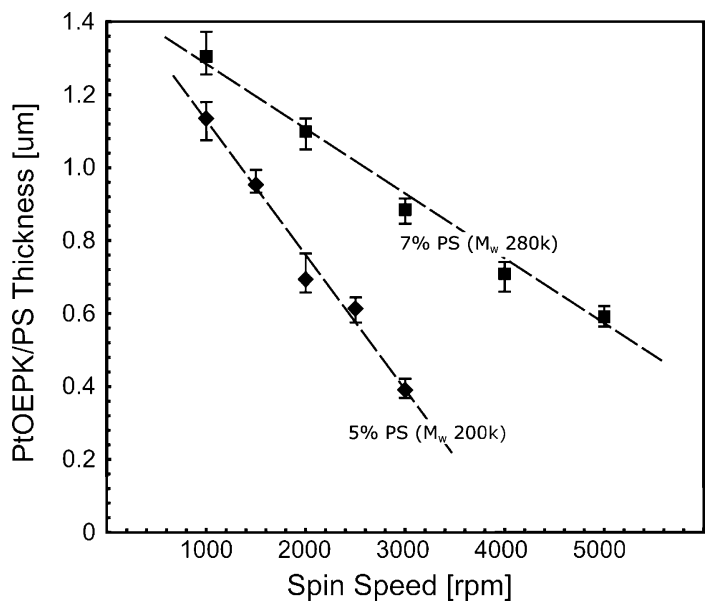

Fig. 2 Plot of PtOEPK/PS sensor film thickness vs. spin speed for two different percentage and molecular weight solutions of PS on glass microscope slides. Spin duration was $30 \mathrm{~s}$ and thickness was measured $24 \mathrm{~h}$ after application.

\section{Sensor patterning}

In case of supported positive stamps, as used in this work, a high etch pressure was chosen to allow for under-etching of the thick stamp support. The unmasked vertical etch rate of PtOEPK/PS in the Oxford Plasmalab 80i reactive ion etcher was determined to be $150 \mathrm{~nm} \mathrm{~min}^{-1}$ at $200 \mathrm{~W}$ power and $50 \mathrm{sccm} \mathrm{O}$ flow rate, compared to $108 \mathrm{~nm} \mathrm{~min}{ }^{-1}$ reported for pure PS $(75 \mathrm{~W}, 30$ sccm). ${ }^{27}$ Removal of PtOEPK/PS underneath the support was found to depend on the feature height of the stamp pattern. For $100 \mu \mathrm{m}$ high features, a support in excess of $5 \mathrm{~cm}$ could be routinely cleared in a $10 \mathrm{~min}$ etch, compared to $7 \mathrm{~min}$ for $200 \mu \mathrm{m}$ features. The process is able to replicate complex patterns with $100 \mu \mathrm{m}$ line width $(25 \mu \mathrm{m}$ minimum feature size $)$ and can be integrated to simultaneously pattern various extracellular matrix proteins. $^{30}$

\section{Device integration}

To demonstrate applicability of the fabrication process to LOC devices, sensor patterns fabricated on glass microscope slides were combined with a fluidic structure in PDMS by oxygen plasma bonding. The fully assembled bioreactor device with external fluidic connections is shown in Fig. 3. Three cell-culture chambers of different shapes, and hence different wall shearstress profiles, are integrated on the chip. The lateral tapering of the middle chamber is specifically designed to generate a linearly increasing wall shear stress along its length. Sensor patterns in the individual chambers are restricted to the indicated outlines by the patterning process described before. A closeup of the middle PDMS chamber is shown in Fig. 3(b). The corresponding sensor pattern realized within the chamber was 

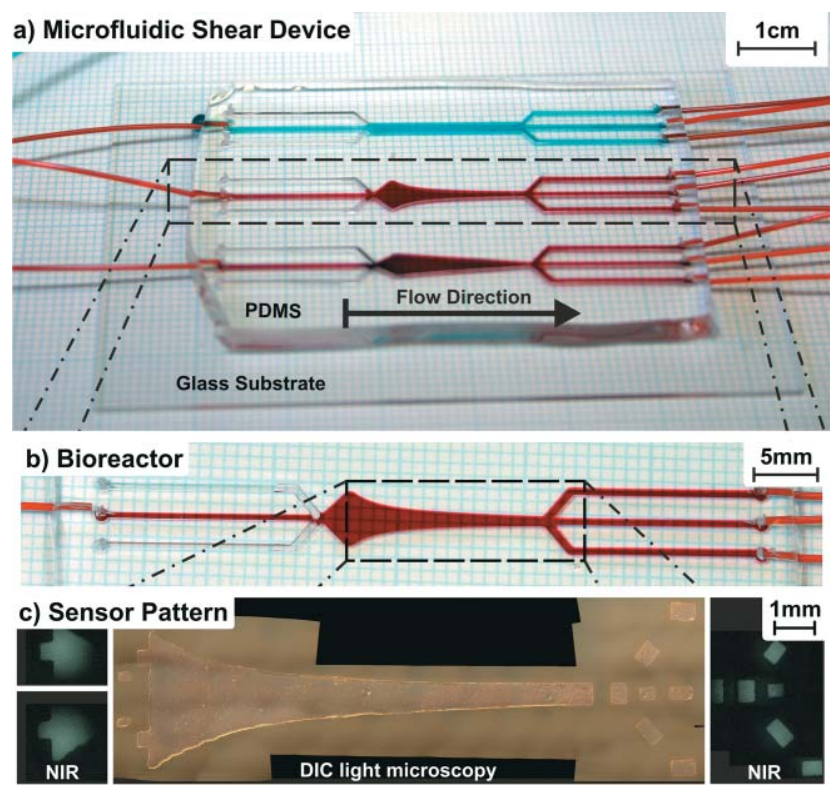

Fig. 3 (a) PDMS-based shear device with integrated oxygen sensors and external interfacing. Red and blue dye in DI water was flowed through the channels to aid visualization and to test fluidic sealing. (b) Close-up showing the central bioreactor device providing linearly increasing shear stress. (c) DIC light microscopy and NIR fluorescence images of the sensor patterns integrated into the bioreactor and inlet/outlet channels.

imaged in multiple, separate DIC images, which were then automatically overlaid to provide the complete micrograph shown in Fig. 3(c). Intensity images on the left and right of Fig. 3(c) show parts of the sensor response in NIR. The large sensor shape inside the chamber and the finer patterns in the in-/outflow channels were fabricated simultaneously with the same PDMS stamp, thus demonstrating the flexibility of the patterning. Since the fabrication process produces very homogenous sensor films we chose to investigate fluorescence intensity quenching, as opposed to luminescent lifetime, for the following sensor characterisation due to its ability to resolve laterally distributed oxygen concentrations.

\section{Sensor optimisation}

After spin-coating and patterning, the sensor signal intensity $I$ of the different films was measured for exposure to partial oxygen pressures of $0 \%\left(I_{0}\right)$ and $100 \%\left(I_{100}\right)$. Intermediate partial pressure values were also measured in order to ascertain the validity of the linear Stern-Volmer relationship ${ }^{31}$

$$
\frac{I_{0}}{I}=\frac{\tau_{0}}{\tau}=1+K_{\mathrm{SV}}^{\mathrm{S}}\left[\mathrm{O}_{2}\right]=1+K_{\mathrm{SV}}^{\mathrm{G}} \mathrm{pO}_{2},
$$

where $I$ is the intensity, $\tau$ the lifetime, $K_{\text {sv }}^{\mathrm{s}}$ and $K^{\mathrm{G}}$ sv are the Stern-Volmer constants for solution and gas, respectively, $I_{0}$ and $\tau_{0}$ are the reference values in the absence of oxygen, $\left[\mathrm{O}_{2}\right]$ is the oxygen concentration in solution and $\mathrm{pO}_{2}$ is the gaseous partial pressure of oxygen. It was observed that PS $\mathrm{M}_{\mathrm{w}}$ and concentration, as well as film thickness, affect the sensor signal intensity ratio $I_{0} / I_{100}$. This ratio, in conjunction with the SternVolmer constant, is commonly used as an indicator of the sensitivity of the sensing film. ${ }^{32,33}$ Sensors with $I_{0} / I_{100}$ larger than 3.0 are considered suitable oxygen-sensing devices. ${ }^{34}$ Fig. 4 plots the intensity ratio as a function of film thickness for the two different PS solutions. As can be seen, the intensity response and thereby sensor sensitivity increases linearly with decreasing film thickness. For the $7 \%$ PS solution $I_{0} / I_{100}$ increases almost two-fold from 3.6 at $1.3 \mu \mathrm{m}$ film thickness to 6.8 at $0.6 \mu \mathrm{m}$. One possible explanation for this increase in the intensity ratio is the increase in the surface area-to-volume ratio. With decreasing film thickness the overall intensity contribution of dye close to the surface will increase, whereas the permeability-limited intensity contribution from dye molecules in the PS bulk is reduced.

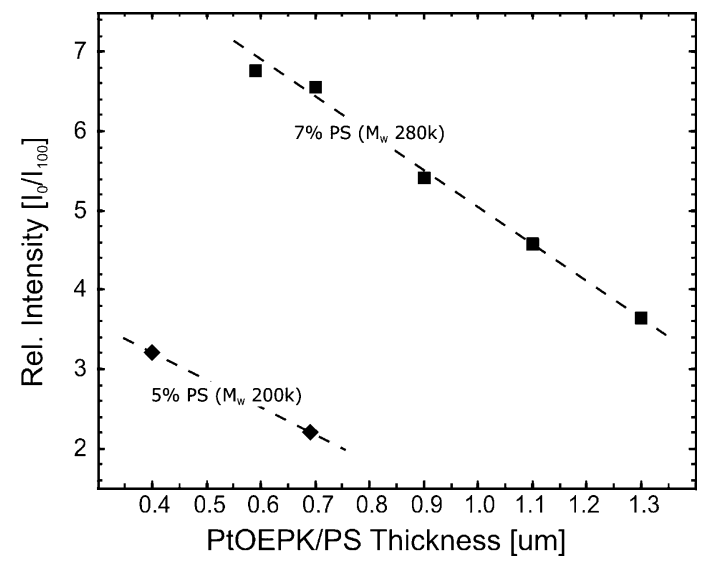

Fig. 4 Plot of the sensor intensity ratio $v s$. film thickness for the two PtOEPK/PS solutions ( $I_{0}$ corresponds to $0 \%$ and $I_{100}$ to $100 \%$ gaseous oxygen, respectively).

The second observation from Fig. 4 is the dependence of the increase in intensity ratio on PS solution. A $0.7 \mu \mathrm{m}$ thick film in $7 \%$ PS solution $\left(\mathrm{M}_{\mathrm{W}}=280000\right)$ shows a three-fold increase in $I_{0} / I_{100}=6.5$ compared to 2.2 for a $5 \%$ PS solution $\left(\mathrm{M}_{\mathrm{W}}=200000\right)$ of equal thickness. We attribute this significant difference mainly to the higher molecular weight PS used in the $7 \%$ solution. During solvent evaporation the system of PS chains strives to attain a state of minimal energy by contraction. Molecules above the entanglement molecular weight of PS $\left(\mathrm{M}_{\mathrm{e}, \mathrm{PS}}=18000\right)$ are thought to contract more slowly with increasing $\mathrm{M}_{\mathrm{W}}$ and to be frozen in place before complete solvent evaporation. ${ }^{35}$ This formation of disordered molecule chains can lead to an increase in oxygen-permeability in PS films of higher $\mathrm{M}_{\mathrm{W}}$. Although preliminary, the above results indicate further possibility for optimisation of the sensor sensitivity through the use of thinner films and PS of different molecular weight. A further possible parameter not investigated here is the influence of the substrate material. While the maximum intensity ratio of 6.8 observed in our experiments is, to our knowledge, the highest reported for PtOEPK/PS on a LOC-compatible glass substrate, even higher values $\left(I_{0} / I_{100}=20\right)$ have been reported for mylar substrates. ${ }^{36}$

\section{Flow characterisation}

Integrated sensor patterns were calibrated for dissolved oxygen measurement in aqueous media by flowing DI water of calibrated oxygen concentration through the device. Sensor response was tested for flow rates from 0.05 to $2 \mathrm{~mL} \mathrm{~min} \mathrm{~m}^{-1}$ 
as corresponding to the shear-stress range of interest in the final device application. ${ }^{21}$ Characterisation experiments were performed with the microfluidic chip equilibrated to a temperature of $37^{\circ} \mathrm{C}$ to ensure compatibility of the sensor test with cellculture conditions. A sensor pattern, as depicted in Fig. 3(c), was fabricated in $1.1 \mu \mathrm{m}$ thick $7 \% \mathrm{w} / \mathrm{w}$ PtOEPK/PS. The higher film thickness was chosen to allow for simultaneous observation of any possible effects of shear stress on film adherence. Sensor patterns were found to remain strongly attached up to the highest flow rate tested $\left(2 \mathrm{~mL} \mathrm{~min}^{-1}\right)$. Intensity images for the calibration were recorded on a rectangular sensor patch at the bioreactor entrance. Fig. 5 shows the calibration results for this sensor with both gaseous and dissolved oxygen. The gaseous sensor response was recorded prior to introducing DI water by blowing air, oxygen and nitrogen through the device. Fig. 5(a) shows the resulting three-point calibration curve for detection of gaseous oxygen. A plot of the dynamic sensor response in Fig. 5(b) demonstrates excellent signal reversibility and a nearly instantaneous sensor response under gas flow.

DO detection was successively calibrated with DI water of five different oxygen concentrations produced by the external oxygenator. Fig. 5(c) shows the calibration plot of relative intensity as a function of DO concentration at a constant flow rate of $1 \mathrm{~mL} \mathrm{~min}{ }^{-1}$. The maximum $\mathrm{DO}$ concentration of $18.4 \mathrm{mg} \mathrm{L}^{-1}$ produced by the oxygenator caused a factor of 3.4 change in total fluorescent intensity. The sensor was found to follow the linear Stern-Volmer model (eqn (1)) with an intensity ratio $I_{0} / I_{100}$ of 3.4 at this flow rate.

Calibration experiments were further extended to determine the dynamic range of the sensor for different flow rates. Fig. 6(d) plots the absolute sensor intensity of oxygenated $\left(\sim 100 \% \mathrm{O}_{2}\right)$ and nitrogenated $\left(\sim 0 \% \mathrm{O}_{2}\right)$ DI water as a function of flow rate. The full-scale operating range of the sensor is reached at

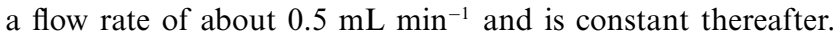
For flow rates below this value the operating range decreases slightly due to parasitic convective losses to the surrounding PDMS. ${ }^{13}$ Depending on the initial concentration entering the device, oxygen is either added or removed from the DI water by mass transfer through the device walls. This effect is due to the high permeability of oxygen in PDMS and can be reduced by use of a less permeable polymer or by encapsulation of the final device. Since the permeation coefficient of $\mathrm{N}_{2}$ in PDMS has been found to be a factor two smaller than that of $\mathrm{O}_{2},{ }^{37}$ the signal decrease for nitrogenated water is larger than for oxygenated at low flow rates.
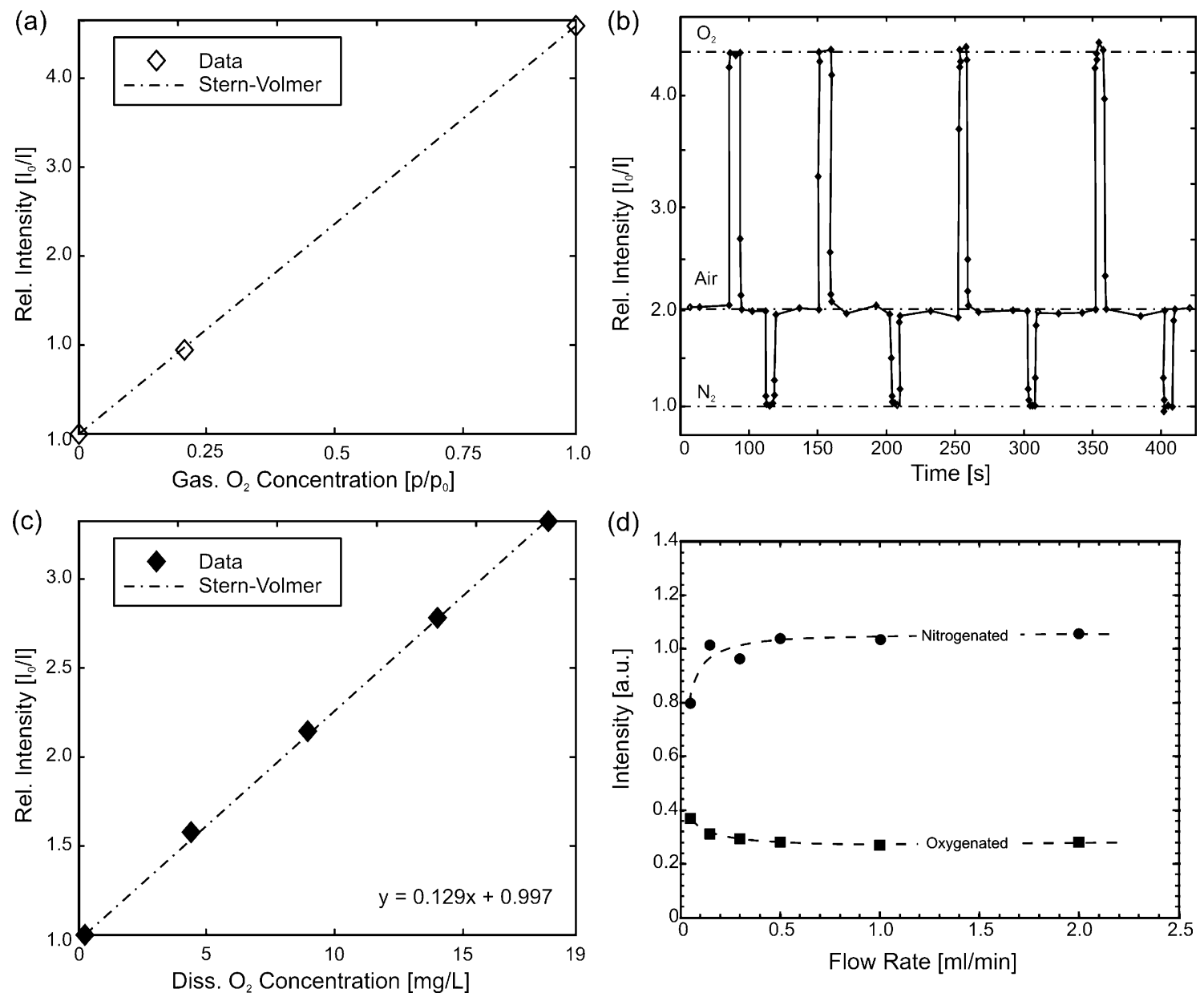

Fig. 5 (a) Three-point calibration curve for detection of gaseous oxygen. (b) Dynamic sensor response (relative intensity, $I_{0} / I$ ) for alternating gaseous oxygen concentrations. (c) Stern-Volmer calibration curve for detection of dissolved oxygen in DI water with the same sensor pattern at a flow rate of $Q=1 \mathrm{~mL} \mathrm{~min}{ }^{-1}$. (d) Plot of the sensor intensity data corresponding to flows of nitrogenated and oxygenated water across the tested dynamic operating range. At low flow rates the operating range decreases slightly due to parasitic convective losses to the surrounding PDMS. 


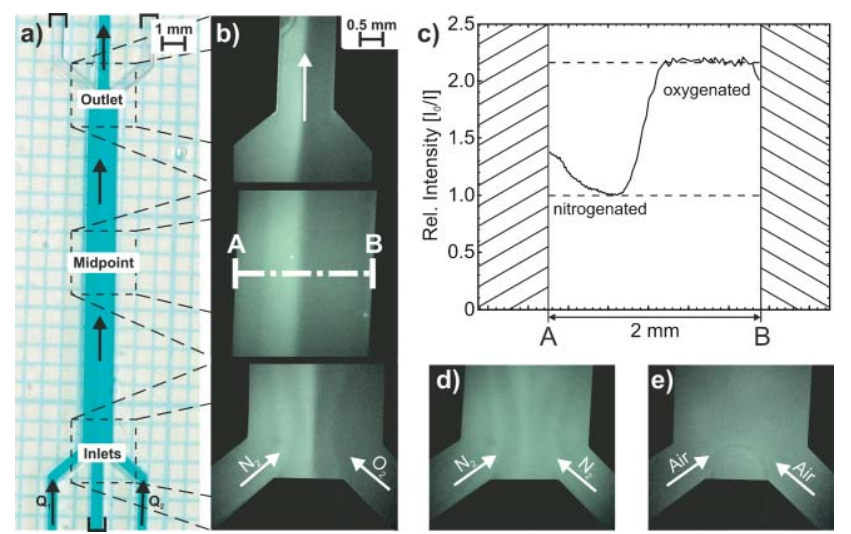

Fig. 6 Detection of laterally varying oxygen concentrations inside a microfluidic device. (a) Photograph of the rectangular bioreactor with integrated oxygen sensor film and filled with coloured DI water for illustration. The two inlets at the bottom were used to provide flows of differing oxygen concentration into the device, $Q_{1}=Q_{2}=500 \mathrm{~mL} \mathrm{~min}^{-1}$. (b) Intensity images for dual flow of nitrogenated $\left(\mathrm{N}_{2}\right)$ and oxygenated $\left(\mathrm{O}_{2}\right)$ water at the device inlet, midpoint and outlet. No convective mixing is observed due to laminar flow conditions. (c) Plot of the relative intensity signal across the reactor width at the midpoint (A-B) showing a clear transition between the two flow lines. (d), (e) Intensity images for the device inlet after switching both inlet flows to nitrogenated and aerated water.

Stability and repeatability of the PtOEPK/PS sensor response was found to be excellent. Dynamic measurements (Fig. 5(b)) were performed repeatedly under $1 \mathrm{~h}$ continuous illumination and showed no significant change in sensor intensity. This duration is sufficient for extensive cell-culture studies with multiple periods of data acquisition per day. Furthermore, 3 weeks of continuous illumination with only minor signal degradation and the retaining of spectral and quenching characteristics for up to 2 years under storage have been reported for PtOEPK/PS. ${ }^{19}$

\section{Sensor application}

Measurement of locally varying oxygen concentrations inside a microfluidic channel is one possible application for the sensors produced by the patterning process. To demonstrate this we applied two inlet flows of differing oxygen concentration to the device and recorded the change in intensity of an integrated sensor film. A set of two identical external oxygenators was used to provide aerated (air), nitrogenated $\left(\mathrm{N}_{2}\right)$ or oxygenated $\left(\mathrm{O}_{2}\right)$ flow of DI water via two inlets. The flow rate of both inlets was kept constant at $0.5 \mathrm{~mL} \mathrm{~min}^{-1}$ by use of a dual syringe pump. The two inlets lead to a rectangular reactor chamber (depth $0.2 \mathrm{~mm}$, width $2 \mathrm{~mm}$, length $18 \mathrm{~mm}$ ) and exit through an outlet at the end of the chamber. Fig. 6 shows a photograph of the device and the corresponding intensity images at the inlet, the midpoint of the reactor and the outlet. For illustration purposes this device was filled with blue dye-coloured DI water. Intensity images at the bottom of Fig. 6 correspond to the sensor signal at the reactor inlet for combinations of nitrogenated-oxygenated (b), nitrogenated-nitrogenated (d) and aerated-aerated (e) water.

As can be observed, no convective mixing takes place over the reactor length due to laminar flow conditions $(\operatorname{Re} \sim 80)$. The nitrogenated and oxygenated flow lines remain parallel and easy to distinguish from reactor inlet to outlet. Fig. 6(c) plots the relative signal intensity across the channel width (A-B) for parallel nitrogenated-oxygenated flows at the reactor midpoint. A clear transition of oxygen concentration between the two flows can be observed along the reactor centreline. The deviation of the plot shape from a stepwise function increases along the reactor length due to diffusive transport of oxygen from the oxygenated flow on the right to the nitrogenated flow on the left. This could potentially be used to determine the constant of diffusion for oxygen in different media. The increase in signal towards the left boundary of the nitrogenated flow was attributed to flow instabilities induced by slight leakage at the tubing-PDMS interface of the left inlet port.

\section{Conclusions}

We have shown the fabrication and characterisation of polymerencapsulated fluorescent dye-based optical oxygen sensors for LOC devices. Sensor films of PtOEPK/PS were applied by spin-coating and the influence of film thickness and molecular weight of the matrix polymer on the fluorescence signal intensity was investigated. Films were patterned by soft lithography and integration into a PDMS-based microfluidic device was demonstrated. Patterned and integrated sensor films were further characterized and calibrated for measurement of gaseous and dissolved oxygen. Characterisation showed excellent intensity ratios for measurement of both gaseous and dissolved oxygen, making them attractive for application in high sensitivity environments. Additionally, the applicability of the sensor fabrication to cell-culture bioreactors, point-of-care diagnostics or LOC devices was demonstrated by detection of laterally varying oxygen concentrations inside a microfluidic device. In general, the process described here allows for automated wafer-level fabrication and integration of sensor patterns into LOC devices, thereby increasing optical signal stability and repeatability. While demonstrated for optical oxygen sensors, the process has the potential to significantly simplify integration of various other polymer-based sensors.

\section{Acknowledgements}

The authors would like to thank Erwin Berthier and Florian L'Hostis for fruitful discussions and Helen Devereux and Gary Turner for technical assistance.

\section{References}

1 G. L. Semenza, Cell, 2001, 107, 1-3.

2 T. Kietzmann and K. Jungermann, Cell Biol. Toxicol., 1997, 13, 243 255.

3 J. W. Allen and S. N. Bhatia, Biotechnol. Bioeng., 2003, 82, 253-262.

4 S. Roy, S. Khanna, A. A. Bickerstaff, S. V. Subramanian, M. Atalay, M. Bierl, S. Pendyala, D. Levy, N. Sharma, M. Venojarvi, A. Strauch, C. G. Orosz and C. K. Sen, Circ. Res., 2003, 92, 264-271.

5 L.-L. Zhu, L.-Y. Wu, D. Yew and M. Fan, Mol. Neurobiol., 2005, 31, 231-242.

6 F. C. O'Mahony, C. O’Donovan, J. Hynes, T. Moore, J. Davenport and D. B. Papkovsky, Environ. Sci. Technol., 2005, 39, 5010-5014.

7 C.-C. Wu, T. Saito, T. Yasukawa, H. Shiku, H. Abe, H. Hoshi and T. Matsue, Sens. Actuators, B, 2007, 125, 680-687.

8 D. A. Chang-Yen and B. K. Gale, Lab Chip, 2003, 3, 297-301.

9 J. Karasinski, L. White, Y. C. Zhang, E. Wang, S. Andreescu, O. A. Sadik, B. K. Lavine and M. Vora, Biosens. Bioelectron., 2007, 22, 2643-2649. 
10 E. Akyilmaz, A. Erdogan, R. Ozturk and I. Yasa, Biosens. Bioelectron., 2007, 22, 1055-1060.

11 J. Alderman, J. Hynes, S. M. Floyd, J. Kruger, R. O'Connor and D. B. Papkovsky, Biosens. Bioelectron., 2004, 19, 1529-1535.

12 D. Sud, G. Mehta, K. Mehta, J. Linderman, S. Takayama and M.-A. Mycek, J. Biomed. Opt., 2006, 11, 050504-050503.

13 A. P. Vollmer, R. F. Probstein, R. Gilbert and T. Thorsen, Lab Chip, $2005, \mathbf{5}, 1059-1066$.

14 P. Roy, H. Baskaran, A. W. Tilles, M. L. Yarmush and M. Toner, Ann. Biomed. Eng., 2001, 29, 947-955.

15 X. Xiong, D. Xiao and M. M. F. Choi, Sens. Actuators, B, 2006, 117, $172-176$.

16 S. Lee, B. L. Ibey, G. L. Cote and M. V. Pishko, Sens. Actuators, B, 2008, 128, 388-398.

17 C. O'Donovan, J. Hynes, D. Yashunski and D. B. Papkovsky, J. Mater. Chem., 2005, 15, 2946-2951.

18 M. Smiddy, N. Papkovskaia, D. B. Papkovsky and J. P. Kerry, Food Res. Intern., 2002, 35, 577-584.

19 D. B. Papkovsky, G. V. Ponomarev, W. Trettnak and P. O'Leary, Anal. Chem., 1995, 67, 4112-4117.

20 V. Nock, R. J. Blaikie, and T. David, in 33rd Int. Conf. Micro \& Nano Eng. MNE, Copenhagen, 2007.

21 V. Nock, R. J. Blaikie and T. David, Proc. SPIE-Int. Soc. Opt. Eng., 2007, 6799, 67990Y-67910.

22 B. R. Flachsbart, K. Wong, J. M. Iannacone, E. N. Abante, R. L. Vlach, P. A. Rauchfuss, P. W. Bohn, J. V. Sweedler and M. A. Shannon, Lab Chip, 2006, 6, 667-674.
23 B. H. Jo, L. M. Van Lerberghe, K. M. Motsegood and D. J. Beebe, J. Microelectromech. Syst., 2000, 9, 76-81.

24 B. Samel, V. Nock, A. Russom, P. Griss and G. Stemme, Biomed. Microdevices, 2007, 9, 61-67.

25 V. Nock, R. J. Blaikie and T. David, N. Z. Med. J., 2007, 120, 2-3.

26 Y. N. Xia and G. M. Whitesides, Angew. Chem., Int. Ed., 1998, 37, $551-575$.

27 G. N. Taylor, T. M. Wolf and J. M. Moran, J. Vac. Sci. Technol., 1981, 19, $872-880$.

28 M. Brown, Autostitch, http://www.cs.ubc.ca/ mbrown/autostitch/ autostitch.html, accessed January, 2007.

29 R. L. Hamilton, M. N. Berry, M. C. Williams and E. M. Severinghaus, J. Lipid Res., 1974, 15, 182-186.

30 S. W. Rhee, A. M. Taylor, C. H. Tu, D. H. Cribbs, C. W. Cotman and N. L. Jeon, Lab Chip, 2005, 5, 102-107.

31 Y. Amao, Microchim. Acta, 2003, 143, 1-12.

32 A. Mills, Sens. Actuators, B, 1998, 51, 60-68.

33 Y. Fujiwara and Y. Amao, Talanta, 2004, 62, 655-660.

34 B. D. MacCraith, C. M. McDonagh, G. O'Keeffe, E. T. Keyes, J. G. Vos, B. O'Kelly and J. F. McGilp, Analyst, 1993, 118, 385388.

35 J. Zhao, S. Jiang, Q. Wang, X. Liu, X. Ji and B. Jiang, Appl. Surf. Sci., 2004, 236, 131-140.

36 P. Hartmann and W. Trettnak, Anal. Chem., 1996, 68, 2615-2620.

37 M. Ohyanagi, H. Nishide, K. Suenaga and E. Tsuchida, Polym. Bull. (Berlin), 1990, 23, 637-642. 